\title{
Results of Combined Small Incision Cataract Surgery and Cyclodialysis in Low Income Patients with Glaucoma and Cataract
}

\author{
Mario Renato Papa-Vettorazzi ${ }^{1 *}$, Claudia María Lopez-Villeda ${ }^{1}$, José \\ Benjamín Cruz-Rordriguez ${ }^{2}$, Gladys Lucía Silva-Linares ${ }^{1}$ and Mariano \\ Yee-Melgar ${ }^{1}$ \\ ${ }^{1}$ Visualiza Clinic, 5ta av. 11-44 Zona 9, Guatemala, Guatemala \\ ${ }^{2}$ University of California, San Diego, CA, USA \\ *Corresponding Author: Mario Renato Papa-Vettorazzi, Visualiza Clinic, 5ta av. \\ 11-44 Zona 9, Guatemala, Guatemala.
}

Received: August 16, 2021

Published: December 14, 2021

(C) All rights are reserved by Mario Renato

Papa-Vettorazzi., et al.

\begin{abstract}
Purpose: To assess the short and long term effect of combined cataract and glaucoma surgery (Small Incision Cataract Surgery [SICS] plus cyclodialysis) in a low income population.

Study design: Retrospective.

Materials and methods: We performed a retrospective analysis of 355 SICS plus cyclodialysis procedures all performed by experienced surgeons. Evaluations at day one, 1 week, 1, 3, 6 and 12 months. At every visit, the evaluation included: intra ocular pressure (IOP) measurement, glaucoma medication requirements and corrected distance visual acuity (CDVA). We also evaluated complications and re interventions.

Results: The median preoperative IOP was $18 \mathrm{mmHg}(15-24)$, it decreased to $12 \mathrm{mmHg}(10-14)$ at the 7 days, 1, 3, 6 and $12 \mathrm{month}$ assessments $(\mathrm{p}<0.01)$. Mean number of glaucoma medications decreased from 1.55 preoperatively to 0.96 postoperatively (mean decrease $0.58,95 \%$ CI-0.84 to -0.32, p < 0.01). At three months median CDVA improved from 0.69 logMar (0.47-1.8) (20/100 Snellen) to $0.3 \log$ Mar (0.17-0.51) (20/40 Snellen) ( $<$ 0.01). Complication rate was $9 \%$, the most frequent was posterior capsule rupture (PCR). Re intervention rate was $10 \%$ mostly because of not achieving IOP control.

Conclusions: Combining SICS plus cyclodialysis is effective, it shows a short and long term reduction in IOP, a decrease in glaucoma medication requirements and an improvement in CDVA all comparable to the reports of phacoemulsification plus trabeculectomy.
\end{abstract}

Keywords: Combined Surgery; Cyclodialysis; Glaucoma Surgery; SICS

\section{Abbreviations}

AGIS: Advanced Glaucoma Intervention Study; AVT: Anterior Vitrectomy SICS: Small Incision Cataract Surgery; CACG: Chronic Angle Closure Glaucoma; CDVA: Corrected Distance Visual Acuity; IN: Inferior Nasal; IOP: Intraocular Pressure; IQR: Interquartile Range; IT: Inferior Temporal; PCR: Posterior Capsule Rupture; POAG: Primary Open Angle Glaucoma; PXG: Pseudo Exfoliation Glaucoma; SD: Standard Deviation; SN: Superior Nasal; ST: Superior Temporal.

\section{Introduction}

Glaucoma is defined as an optic neuropathy caused by a group of diseases than can be associated with a rise in intraocular pressure (IOP). Despite all the technologic advances, glaucoma is still a major public health problem (second cause of blindness according to the World Health Organization) [1], especially in low income countries where access to specialized healthcare services and cost of treatment represent an important barrier. 
It is common that patients with advanced glaucoma with surgical indication, also presents with cataract. In these cases, the two procedures can be performed combined or sequentially. The question about surgical order is widely debated. The decision is made according to clinical findings and possible advantages of the approach [2].

It is known that combined phaco-trabeculectomy allow for greater IOP reduction and fewer IOP spikes in the immediate postoperative period [2]. However in low income patients this type of surgery has certain disadvantages mainly according to cost and follow up, that is why Small Incision Cataract Surgery (SICS) plus cyclodialysis can be an effective alternative. There are some reports concluding that the combination of cataract extraction plus cyclodialysis is successful in lowering both IOP and postoperative glaucoma medications [3-5].

The purpose of this study was to evaluate the results and determine the effectiveness of combined SICS plus cyclodialysis, and to compare these results with phaco-trabeculectomy publications. The main objective was to assess the reduction on IOP and secondary objectives were: glaucoma medication requirements, change in best corrected distance visual acuity (CDVA) at 3 months, complications and re interventions.

\section{Study design}

Single center, retrospective cohort study, including 355 eyes that underwent SICS plus cyclodialysis.

\section{Materials and Methods}

All procedures were performed by experienced surgeons using a standard technique during 2017 and 2018. Every eye had a significant visual impairing cataract and advanced uncontrolled glaucoma or uncontrolled ocular hypertension (IOP higher than the considered target for each case despite medical or laser therapy [according to clinical evaluation]). A/B scan was performed prior to surgery in all patients with mature cataract that did not allowed fundoscopic evaluation.

Optic nerve damage consideration and glaucoma classification was made based on clinical evaluation and preoperative IOP.

Manual chart review was performed by one of the investigators and validated by other. Clinical variables extracted were: age, sex, eye, type of glaucoma and excavation (clinical evaluation). IOP and number of glaucoma medications, both preoperatively and postoperatively at day one, 1 week, 1, 3, 6 and 12 months. Preoperative and postoperative CDVA at 3 months.
An external Institutional Ethics Committee confirmed the exempt status of this work given its retrospective nature and approved the study. Research adhered to the tenets of the Declaration of Helsinki.

Because some visual acuities were expressed in non-numerical terms, they were assigned a rank/value in logMar scale for their analysis (Appendix A).

\begin{tabular}{|l|c|}
\hline Snellen & LogMar \\
\hline $20 / 20$ & 0.00 \\
$20 / 25$ & 0.09 \\
$20 / 30$ & 0.17 \\
$20 / 40$ & 0.30 \\
$20 / 50$ & 0.39 \\
$20 / 60$ & 0.47 \\
$20 / 70$ & 0.54 \\
$20 / 80$ & 0.60 \\
$20 / 100$ & 0.69 \\
$20 / 140$ & 0.84 \\
$20 / 200$ & 1.00 \\
$20 / 400$ & 1.30 \\
CD & 1.80 \\
MM & 1.90 \\
PL & 2.00 \\
NPL & 2.50 \\
\hline
\end{tabular}

Appendix A: Visual acuity, assigned values for statistical analysis.

$$
\begin{gathered}
\text { CD: Counting Fingers } \\
\text { MM: Hand Movement } \\
\text { PL: Light Perception } \\
\text { NPL: No Light Perception. }
\end{gathered}
$$

\section{Surgical procedure}

After sedation administered by an anesthesiologist and peribulbar anesthesia application (1:1 mix of $1 \%$ lidocaine, $0.75 \%$ bupivacaine +150 units of hyaluronidase). A lid speculum was placed in the eye, a $6 \mathrm{~mm}$ fornix-based peritomy was performed followed by light wetfield diathermy on episcleral vessels. A 5.5 - $6 \mathrm{~mm}$ frown scleral incision with a diamond blade or a $15^{\circ}$ scalpel was created starting 1 - $2 \mathrm{~mm}$ behind the limbus (location of both peritomy and scleral incision was decided by the surgeon according to the preoperative keratometry). This incision was carried forward with a crescent blade until the limbus was reached advancing it $1-1.5$ $\mathrm{mm}$ into clear cornea. A paracentesis with a $3.2 \mathrm{~mm}$ keratome blade was performed. In some cases, surgeons used trypan blue to dye the anterior capsule, then the anterior chamber was filled with viscoelastic. Subsequently, the entire internal lip of the tun- 
nel incision was opened in a valved fashion with the $3.2 \mathrm{~mm}$ keratome blade. Following this, a capsular rhexis using a cystotome was performed. Hydrodissection and cataract luxation to the anterior chamber for posterior cataract extraction using an irrigation vectis was made. All cortical material was removed using a 21 simcoe cannula. Then a single piece polimethylmetracylate lens was introduced in the capsular bag. After this, a miotic was used and a 2 hour cyclodialysis was performed (location of the cyclodialysis was determined by the surgeon).

To create the cyclodialysis, a cyclodialysis spatula was introduced through the paracentesis at the opposite quadrant where it would be performed. An initial superficial movement was made to perform a goniotomy. Following this, while maintaining a light upward lift on the sclera, the peripheral cornea was followed with the spatula to reach the scleral spur. Then the tip of the spatula was gently encouraged and allowed to pass beyond the scleral spur into de supracilliary space. (Appendix B).

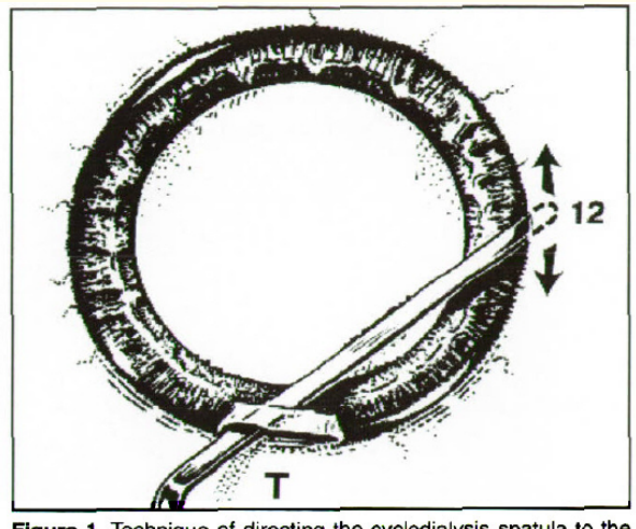

Figure 1. Technique of directing the cyclodialysis spatula to the 12-0'clock angle through a temporal corneal incision.

Appendix B. Cyclodialysis cleft procedure [4].

Image taken from Rowan PJ. Combined Cyclodialysis and Cataract Surgery. Ophthalmic Surg Lasers. 1998;29(12): 962-968.

Once the cyclodialysis was created, the anterior chamber was observed during 1 or 2 minutes for any significant hemorrhage, then the viscoelastic was removed, the paracentesis hydrated and the main wound checked for any leakage. Finally, the conjunctiva was closed using either diathermy or a 10-0 nylon suture.

All eyes were patched and shielded. Every patient used combined antibiotic and steroid drops every 1 or 2 hours while awake for 7 days, then tapered slowly for 1 month unless inflammation did not resolve. In some cases, pilocarpine $4 \%$ was used 3 times a day to induce miosis and avoid the cyclodialysis cleft closure.

\section{Postoperative care}

Follow up was at day one, then every week for the first month and then at 3, 6 and 12 months.

Definition of success and qualified success after glaucoma surgery is not universal [6]. The most frequent definition uses a target IOP of $<18 \mathrm{mmHg}$ to define success. However, we consider that in eyes with advanced glaucoma the aim should be to obtain lower IOP levels.

The Advanced Glaucoma Intervention Study (AGIS) has suggested that an IOP at which visual field loss stabilizes is an average of $12.3 \mathrm{mmHg}$ and that the aim should be to obtain a $40 \%$ reduction from baseline in severe cases and 30\% reduction from baseline in moderate cases $[2,7]$.

That is why we performed 2 separate analyses. One applying the definition of IOP < $18 \mathrm{mmHg}$ :

- Success: IOP < $18 \mathrm{mmHg}$ without glaucoma medication.

- Qualified success: IOP $<18$ mmHg with glaucoma medication.

- $\quad$ Failure: IOP $\geq 18 \mathrm{mmHg}$ or need of subsequent procedures to control IOP.

And the other, applying the definition suggested by AGIS $[2,6]$ :

- $\quad$ Success: IOP $\leq 12.3 \mathrm{mmHg}$ or a $40 \%$ reduction from baseline in severe cases and $30 \%$ reduction from baseline in moderate cases, without glaucoma medication.

- $\quad$ Qualified success: IOP $\leq 12.3 \mathrm{mmHg}$ or a $40 \%$ reduction from baseline in severe cases and 30\% reduction from baseline in moderate cases, with glaucoma medication.

- Failure: IOP > $12.3 \mathrm{mmHg}$ or less than $40 \%$ reduction from baseline in severe cases and less than $30 \%$ reduction from baseline in moderate cases.

We also evaluated glaucoma medication requirements, change in CDVA at 3 months, complications and re-interventions.

\section{Statistical analysis}

Descriptive analysis was made for all the continuous variables. Given the small sample size, normality of the variables was evaluated with Shapiro-Wilk test and visually with a Q-Q plot. The nor- 
mally distributed variables are presented with mean and standard deviation (SD), the ones that were not normally distributed are presented with median and interquartile range (IQR). For all the categorical variables we present frequencies and proportions. According to data distribution, paired t-test or non-parametric Kruskal-Wallis test were used to compare continuous variables. The categorical results were analyzed by chi-square test or Fisher exact when the sample was smaller. $P$ values of 0.05 or less were considered statistically significant. Kaplan-Meier survival analysis was made for both definitions of success. Statistical analysis was based on a two sided significance level. Missing data was handled with available data method. Analysis were made using STATA version 14.2 (StataCorp LCC, Tx, EE.UU.).

\section{Results}

SICS plus cyclodialysis was performed in 355 cases, minimum follow up of 7 days, maximum of 12 months, $87 \%$ completed 1 month follow up, $65 \%$ accomplished at least a 3 month follow up, $43 \%$ a 6 month follow up and $30 \%$ completed a 12 month follow up. Loss of follow up was important and was mainly due to patient individual economic reasons, including travel. Of the 355 cases, $70 \%$ were classified as severe glaucoma, $21 \%$ as moderate glaucoma and $9 \%$ as ocular hypertension. Demographic characteristics are presented on table 1.

\begin{tabular}{|l|c|}
\hline Variable & n (\%) \\
\hline Age, median (IQR) & $75(67,80)$ \\
\hline Male & $159(44.8 \%)$ \\
\hline Right eye & $176(49.6 \%)$ \\
\hline Excavation, median (IQR) & $.85(.7, .9)$ \\
\hline Type of glaucoma & \\
POAG & $159(47.9 \%)$ \\
CACG & $95(28.6 \%)$ \\
PXG & $49(14.8 \%)$ \\
Other & $29(8.7 \%)$ \\
\hline Cyclodialysis localization & \\
SN & $213(61.0 \%)$ \\
IN & $67(19.2 \%)$ \\
ST & $29(8.3 \%)$ \\
IT & $40(11.5 \%)$ \\
\hline Preoperative CDVA logMar, median (IQR) & $.69(.47,1.8)$ \\
\hline Preoperative medication, mean (SD) & $1.55(1.40)$ \\
\hline
\end{tabular}

\begin{tabular}{|l|l|}
\hline Preoperative IOP, median (IQR) & $18(15,24)$ \\
\hline Postoperative BCVA logMar, $\mathrm{n}=231$ median & $.3(.17, .51)$ \\
(IQR) & IQR: interquartile range, SD: standard deviation. \\
POAG: primary open angle glaucoma, CACG: & \\
chronic angle closure glaucoma, PXG: pseudo & \\
exfoliation glaucoma, Other: ocular hyperten- & \\
sion, steroid induced glaucoma, combined & \\
mechanism glaucoma, pigmentary glaucoma. & \\
SN: superior nasal, IN: inferior nasal, ST: su- & \\
perior temporal, IT: inferior temporal. CDVA: & \\
best corrected visual acuity, IOP: intraocular & \\
pressure. & \\
\hline
\end{tabular}

Table 1: Demographic characteristics, $n=355$.

Preoperative and postoperative IOP are presented in figure 1. On average the percentage of IOP reduction was $28.6 \%$.

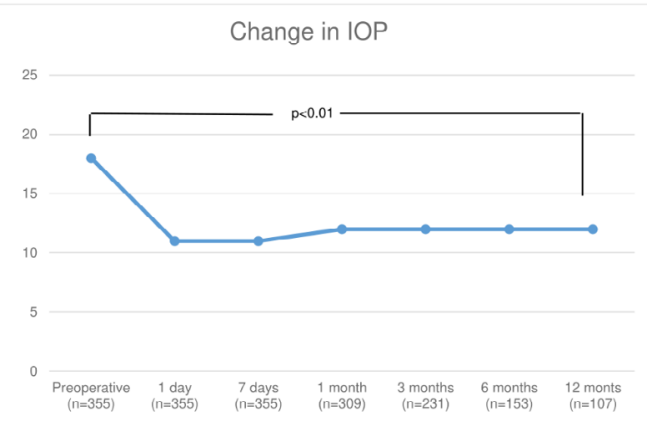

Figure 1: Preoperative and postoperative IOP values. Median preoperative IOP was $18 \mathrm{mmHg}(15-24)$, there was a sustained and significant decrease to $12 \mathrm{mmHg}(10-14)$ at the 7 day, 1 ,

3,6 and 12 month assessments ( $<<0.01$ ). IOP: intraocular pressure. $\mathrm{P}$ value represents t-test for 12 months compared to baseline.

Preoperative and postoperative glaucoma medication requirements can be seen in table 2 . Mean decrease at 12 months was 0.58 medications, $95 \% \mathrm{CI}-0.84$ to -0.32 , $\mathrm{p}<0.01$.

The relationship between location of the cyclodialysis and reduction in IOP and glaucoma medication requirements is presented 


\begin{tabular}{|l|c|c|}
\hline & N & Mean (SD) \\
\hline Preoperatively & 355 & $1.55(1,4)$ \\
\hline 1 month & 309 & $0.52(1.04)$ \\
\hline 3 months & 231 & $0.86(1.12)$ \\
\hline 6 months & 153 & $0.95(1.25)$ \\
\hline 12 months & 107 & $0.96(1.22)$ \\
\hline SD: standard deviation. & & \\
\hline
\end{tabular}

in table 3. For the superior quadrants we obtained a $56.2 \%$ success rate and $84.7 \%$ qualified success rate. For the inferior quadrants a $63.5 \%$ success rate and $89.7 \%$ qualified success rate (IOP $\leq 18$ mmHg). Applying the definition suggested by AGIS, we observed a $45.5 \%$ success rate and a $71.1 \%$ qualified success rate for the superior quadrants. And a $62.6 \%$ success rate and $88.7 \%$ qualified success rate for the inferior quadrants.

Table 2: Number of glaucoma medications used preoperatively and postoperatively.

\begin{tabular}{|c|c|c|c|c|c|c|}
\hline $\begin{array}{l}\text { Variable } \\
\mathbf{N}\end{array}$ & & $\begin{array}{c}\text { SN } \\
213\end{array}$ & $\begin{array}{l}\text { IN } \\
67\end{array}$ & $\begin{array}{l}\text { ST } \\
29\end{array}$ & $\begin{array}{l}\text { IT } \\
40\end{array}$ & $P$ value \\
\hline \multirow{4}{*}{ Type of glaucoma } & POAG & $92(46.2 \%)$ & $32(50.8 \%)$ & $12(46.2 \%)$ & $21(53.8 \%)$ & \multirow{4}{*}{0.36} \\
\hline & CACG & $62(31.2 \%)$ & $16(25.4 \%)$ & $7(26.9 \%)$ & 7 (17.9\%) & \\
\hline & PXG & $27(13.6 \%)$ & $12(19.0 \%)$ & $2(7.7 \%)$ & $8(20.5 \%)$ & \\
\hline & Other & $18(9 \%)$ & $3(4.8 \%)$ & $5(19.2 \%)$ & $3(7.7 \%)$ & \\
\hline $\begin{array}{l}\text { Preoperative IOP } \\
n=355\end{array}$ & Median (IQR) & $19(16,24)$ & $18(16,24)$ & $18(16,24)$ & $17.5(14,23)$ & 0.45 \\
\hline $\begin{array}{l}\text { Preoperative med } \\
n=355\end{array}$ & Median (IQR) & $2(0,3)$ & $1(0,2)$ & $1(0,3)$ & $2(0,3)$ & 0.15 \\
\hline $\begin{array}{l}\text { IOP } 1 \text { day } \\
n=355\end{array}$ & Median (IQR) & $11.5(8,16)$ & $10(8,14)$ & $11(10,15)$ & $9.5(7.5,12)$ & 0.049 \\
\hline $\begin{array}{l}\text { IOP } 7 \text { days } \\
\mathrm{n}=355\end{array}$ & Median (IQR) & $12(9,15)$ & $10(8,15)$ & $10.5(8,13)$ & $10.5(6,13)$ & 0.030 \\
\hline $\begin{array}{l}\text { IOP } 1 \text { month } \\
n=309\end{array}$ & Median (IQR) & $13(10,15)$ & $10(8,14)$ & $12(10,15)$ & $11(8,14)$ & 0.007 \\
\hline $\begin{array}{l}\text { Med } 1 \text { month } \\
n=309\end{array}$ & Median (IQR) & $0(0,0)$ & $0(0,0)$ & $0(0,0)$ & $0(0,0)$ & 0.78 \\
\hline $\begin{array}{l}\text { IOP } 3 \text { months } \\
n=231\end{array}$ & Median (IQR) & $12(10,15)$ & $10(7,13)$ & $12.5(11,14)$ & $10(8,14)$ & $<0.001$ \\
\hline $\begin{array}{l}\text { Med } 3 \text { months } \\
n=231\end{array}$ & Median (IQR) & $0(0,2)$ & $0(0,2)$ & $0(0,2)$ & $0(0,2)$ & 0.91 \\
\hline $\begin{array}{l}\text { IOP } 6 \text { months } \\
\mathrm{n}=153\end{array}$ & Median (IQR) & $12(10,14)$ & $11(8,14)$ & $14(8,16)$ & $10.5(7.5,13,5)$ & 0.15 \\
\hline $\begin{array}{l}\text { Med } 6 \text { months } \\
n=153\end{array}$ & Median (IQR) & $0(0,2)$ & $0(0,2)$ & $1(0,2)$ & $0(0,2)$ & 0.91 \\
\hline
\end{tabular}




\begin{tabular}{|l|c|c|c|c|c|c|}
\hline $\begin{array}{l}\text { IOP } 12 \text { months } \\
\mathrm{n}=107\end{array}$ & Mean (SD) & $12.96(4.63)$ & $9.5(3.44)$ & $13(3.46)$ & $12.25(2.76)$ & 0.017 \\
\hline $\begin{array}{l}\text { Med } 12 \text { months } \\
\mathrm{n}=107\end{array}$ & Median (IQR) & $0(0,2)$ & $0(0,1)$ & $1(0,3)$ & $0(0,2)$ & 0.34 \\
\hline $\begin{array}{l}\text { SN: superior nasal, IN: inferior nasal, ST: superior temporal, IT: inferior temporal. POAG: primary open } \\
\text { angle glaucoma, CACG: chronic angle closure glaucoma, PXG: pseudo exfoliation glaucoma, Others: } \\
\text { ocular hypertension, steroid induced glaucoma, combined mechanism glaucoma, pigmentary glaucoma. } \\
\text { Med: medications, IOP: intraocular pressure. IQR: interquartile range, SD: standard deviation. }\end{array}$ \\
\hline
\end{tabular}

Table 3: Preoperative and postoperative IOP and number of glaucoma medications according to cyclodialysis location.

Complications and re interventions can be seen in table 4. Most of the complications were related to the cataract surgery, not to the cyclodialysis cleft. In this study the re intervention rate was $10 \%$ most re interventions were procedures to control IOP.

\begin{tabular}{|l|c|}
\hline Complications & $23(6.4 \%)$ \\
PCR + AVT & $10(2.8 \%)$ \\
Hyphema (that required treatment) & $1(.28 \%)$ \\
Hypotony & $1(.28 \%)$ \\
Others & $23(6.4 \%)$ \\
\hline Re-interventions & $10(2.8 \%)$ \\
Procedures to control IOP & $4(1.1 \%)$ \\
Anterior chamber washout & \\
Others & \\
\hline PCR + AVT: posterior capsule rupture + anterior & \\
vitrectomy. IOP: intraocular pressure. & \\
\hline
\end{tabular}

Table 4: Complications and re interventions, $\mathrm{n}=355$.

Overall success and qualified success rates applying both definitions are presented in table 5. Kaplan-Meier survival analysis can be seen in figure 2 . Applying the $<18 \mathrm{mmHg}$ definition, mean survival time was $805 \pm 30$ days (2.2 years) and applying AGIS definition, mean survival time was $604 \pm 30$ days (1.65 years).

\begin{tabular}{|c|c|c|}
\hline & IOP $<\mathbf{1 8 m m H g}$ & AGIS (IOP<12.3mmHg) \\
\hline Success & $58.3 \%$ & $52.4 \%$ \\
\hline $\begin{array}{c}\text { Qualified } \\
\text { success }\end{array}$ & $86.1 \%$ & $75.7 \%$ \\
\hline
\end{tabular}

Table 5: Success and qualified success rates. $\mathrm{n}=355$.

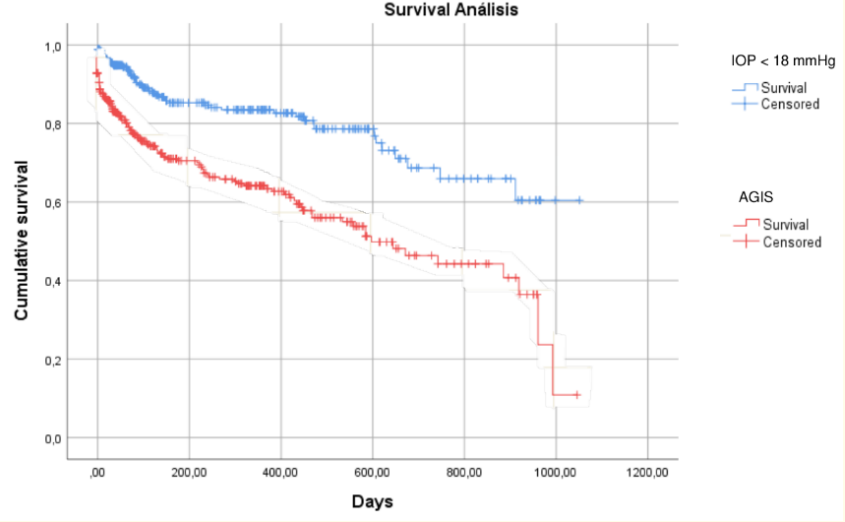

Figure 2: Kaplan-Meier survival analysis applying both definitions. IOP < $18 \mathrm{mmHg}$ and AGIS. Mean survival time was 2.2 years with the $<18 \mathrm{mmHg}$ definition. Applying AGIS criteria, mean survival time was 1.65 years.

\section{Discussion}

Defining success in IOP reduction ideally should involve an assessment of visual field and optic disc stability because the aim ultimately is to protect the structure and function of the optic nerve. A major problem with this is the time required, and the frequency with which testing should be done to detect progression. To overcome this problem, IOP control is often used as a surrogate measure of disease stability and success in studies of glaucoma surgery. However, there is not a clear and universally accepted definition for success and qualified success [6]. 
AGIS has suggested that an IOP at which visual field loss stabilizes is an average of $12.3 \mathrm{mmHg}$ and that the aim should be to obtain a $40 \%$ reduction from baseline in severe cases and $30 \%$ reduction from baseline in moderate cases $[2,7]$.

Currently, the gold standard to treat patients with advanced progressive glaucoma that cannot be controlled by noninvasive therapies is to perform a trabeculectomy with antimetabolite [8]. However, the treatment and follow up are challenging. Success and efficacy are related directly with good post-operative care, frequent appointments and usually minor procedures to modulate and control scarring. All these circumstances translate to overall higher costs [9].

Cyclodialysis as a surgical procedure to treat glaucoma was first described by Heine in 1905 . However, it did not become popular and there are few studies published on this matter. This procedure has some advantages over the trabeculectomy technique: small learning curve, time needed for the procedure is only a few minutes, no need for specialized equipment, no conjunctival manipulation, no need for frequent follow up and low cost [10].

In patients with cataract and uncontrolled glaucoma/ocular hypertension, combined phaco-trabeculectomy is the preferred choice of many surgeons [2]. The reports on success rates for this procedure vary from 67 to $78 \%$ (IOP $<21 \mathrm{mmHg}$ ) [11,12] and qualified success range from 74 to $85 \%$. (IOP $<21 \mathrm{mmHg}$ ) [12,13]. In these studies follow up ranges from 1-42 months.

There are few reports of combined cataract surgery with cyclodialysis. Success rates with this procedure vary from 60 to $83 \%$ and a qualified success range from 72 to $90 \%$ (IOP < $18 \mathrm{mmHg}$ or 20 $\mathrm{mmHg}$ ] [3-5]. In these studies follow up period ranges from 2-34 months.

When we compared our results with the existing literature, using as reference the strictest IOP objective found (IOP $<18 \mathrm{mmHg}$ ) [3], we obtained a $58.3 \%$ success rate and an $86.1 \%$ qualified success rate. These results are similar and comparable with the reports of phaco-trabeculectomy.

However, we considered that in patients with advanced glaucoma the aim should be to obtain lower IOP levels. That is why we applied the definition suggested by AGIS: with this definition, our success rate was $52.4 \%$ and our qualified success was $75.7 \%$.
This study showed a significant reduction in IOP. Median preoperative IOP was $18 \mathrm{mmHg}$ (15-24), and there was a sustained decrease to $12 \mathrm{mmHg}(10-14)$ at the 7 day, 1, 3, 6 and 12 month assessments $(p<0.01)$. On average the percentage of IOP reduction was $28.6 \%$, this is similar to the reports of Ting, Ruderman and Mamalis 27\%, 34\% and 30.8\% (phaco-trabeculectomy) [11,14,15]. And is higher than the $12.65 \%$ reported in the meta analysis published by Jiang (phaco-trabeculectomy) [13].

Reduction in glaucoma medication requirements at 12 months was 0.58 medications, $95 \% \mathrm{CI}-0.84$ to -0.32 , $\mathrm{p}<0.01$. This is lower than the 1.55 reduction reported by Jiang and the 1.44 reported by Stark and Mamalis (phaco-trabeculectomy) [11,13,15].

In our cohort, $61 \%$ of patients needed at least 1 medication before surgery. After the procedure $58 \%$ of patients kept an IOP $<18$ mmHg without medication. These results can be compared with those of Ruderman $63 \%$ but are lower than the reports of Stark $78 \%$ and Mamalis 71.2\%. (phaco-trabeculectomy) $[11,15,16]$.

Applying the definition suggested by AGIS, we observed that $52 \%$ of patients did not required medication to maintain an IOP $<12.3 \mathrm{mmHg}$. Furthermore, 75\% of patients did achieved those goals requiring at least 1 medication.

The reason why CDVA was only described at 3 months is that at the 3 month follow up, every patient had an objective and manifest refraction, therefore a CDVA measurement. CDVA remained unchanged or improved in $90 \%$ of the cases. Median CDVA improved from 0.69 logMar (0.47-1.8) (20/100 Snellen) to 0.3 logMar (0.170.51) (20/40 Snellen) ( $p<0.01)$. This compares to the results published by Stark: $93 \%$ of patients with improvement on CDVA. (phaco-trabeculectomy) [16].

In our cohort, $61 \%$ of patients achieved a CDVA of $0.3 \log$ Mar or better which is comparable to the $63 \%$ reported by Mamalis (phaco-trabeculectomy) [11] and the 64\% reported by Shields (extracapsular extraction plus cyclodialysis) [3].

As reported by Shields, concordant to our data, glaucoma type has no influence on the combined surgery results [3]. However, it is important to acknowledge that cyclodialysis is not recommended as a therapeutic option in patients with neovascular glaucoma, uveitic glaucoma or in presence of important gonioscopic synechiae. 
We considered the relationship between the location of the cyclodialysis and the reduction in IOP and medication requirements. For the superior quadrants we obtained a 56.2\% success and $84.7 \%$ qualified success rate. For the inferior quadrants a $63.5 \%$ success and $89.7 \%$ qualified success rate (IOP $<18 \mathrm{mmHg}$ ).

Overall, the best results were obtained when the cyclodialysis was performed at the inferior nasal quadrant with a qualified success rate of $91 \%$ (applying both criteria, IOP < $18 \mathrm{mmHg}$ and AGIS). This results are comparable to the ones reported by Shields; $87 \%$ of qualified success rate in the inferior quadrants. (Extracapsular extraction plus cyclodialysis) [3].

Complications occurred in $9 \%$ of the patients. Most of the complications were related to the cataract surgery, (posterior capsule rupture [PCR] 6.4\%). Most of the PCR occurred in patients with pseudo exfoliation glaucoma (PXG), hyper mature cataracts and known zonular weakness. This is higher than the $2.8 \%$ PCR rate reported by Shields., et al. however, they excluded eyes with known zonular weakness from their analysis [6]. When excluding eyes with known zonular weakness from our analysis, the PCR rate in our cohort decreases to $2.9 \%$ which is similar to the report from Shields [3].

In this study the re-intervention rate was $10 \%$. Most re-interventions were procedures to control IOP, only 1 patient with severe hypotony that did not responded to noninvasive measures was found. Surgical closure of the cyclodialysis cleft was indicated. We did not find re intervention rates reported on the literature to compare our results.

This study is not free of limitations. Sample size and restricted follow up limited the statistical power of the results. As we collected data from a single center facility and because missing data was handled with available data method some selection bias could be present (if missing pattern was not completely random, however, missing data was less than 5\%). Loss of follow up limits the extrapolation to the long term, although this would bias the results toward the null. The lack of a control group limits our capacity to draw firm conclusions about efficacy. Data was analyzed for changes in each intervened eye over time, and although paired analyses were used, unmeasured confounding could exist. Moreover, optic nerve damage consideration and glaucoma classification was made based on clinical evaluation and preoperative IOP (not visual field damage nor optical coherence tomography of the optic nerve). We believe that further studies including randomized blind interventions should be performed to allow adjustment for other confounders and draw causal conclusions.

\section{Conclusions}

Combined SICS plus cyclodialysis is effective and comparable to phaco-trabeculectomy in terms of success rates, IOP control, medication requirements and CDVA. It is a low cost procedure that can be useful in countries with low income population, it has a relatively easy follow up and a small re intervention rate.

\section{Acknowledgements}

None.

\section{Conflict of Interest}

No conflicting relationship exists for any author.

\section{Bibliography}

1. Bourne Rupert R A., et al. "Causes of vision loss worldwide, 1990-2010: a systematic analysis". The Lancet Global Health 1.6 (2013): e339-349.

2. European Glaucoma Society Terminology and Guidelines for Glaucoma, 4th Edition (2017): 131-191.

3. Shields M B and R J Simmons. "Combined cyclodialysis and cataract extraction”. Ophthalmic Surgery 7.2 (1976): 62-73.

4. Rowan P J. "Combined cyclodialysis and cataract surgery". Ophthalmic Surgery and Lasers 29.12 (1998): 962-968.

5. Hansen S and A B Laursen. "Visualized cyclodialysis--an additional option in glaucoma surgery". Acta Ophthalmologica 64.2 (1986): 142-145.

6. Rotchford Alan P and Anthony J King. "Moving the goal posts definitions of success after glaucoma surgery and their effect on reported outcome". Ophthalmology 117.1 (2010): 18-23.e3.

7. The Advanced Glaucoma Intervention Study (AGIS): 7. The relationship between control of intraocular pressure and visual field deterioration. The AGIS Investigators. American Journal of ophthalmology 130.4 (2000): 429-440.

8. Sanidad MDE and Igualdad SSE. Guía de Práctica Clínica Sobre Glaucoma de Ángulo Abierto. Catalunya (2017). 
9. Urcelay JL., et al. "Trabeculectomía”. Ann d'Oftalmología 23.4 (2015): 52-60.

10. Yee MM. Terapias alternativas para el tratamiento del Glaucoma | VISIÓN (2020).

11. Mamalis N., et al. "Combined phacoemulsification, intraocular lens implantation, and trabeculectomy". Journal of Cataract and Refractive Surgery 22.4 (1996): 467-473.

12. Shingleton Bradford J., et al. "Combined cataract and trabeculectomy surgery in eyes with pseudoexfoliation glaucoma". Journal of Cataract and Refractive Surgery 37.11 (2011): 1961-1970.

13. Jiang Nan., et al. "Meta-analysis of the efficacy and safety of combined surgery in the management of eyes with coexisting cataract and open angle glaucoma". International Journal of Ophthalmology 11.2 279-286.

14. Ting Jessica L M., et al. "Ab interno trabeculectomy: outcomes in exfoliation versus primary open-angle glaucoma". Journal of Cataract and Refractive Surgery 38.2 (2012): 315-323.

15. Ruderman J M., et al. "Combined phacoemulsification and trabeculectomy with mitomycin-C". Journal of Cataract and Refractive Surgery 22.8 (1996): 1085-1090.

16. Stark W J., et al. "The safety and efficacy of combined phacoemulsification and trabeculectomy with releasable sutures". The British Journal of Ophthalmology 90.2 (2006): 146149.

\section{Assets from publication with us}

- Prompt Acknowledgement after receiving the article

- Thorough Double blinded peer review

- Rapid Publication

- Issue of Publication Certificate

- High visibility of your Published work

Website: www.actascientific.com/

Submit Article: www.actascientific.com/submission.php

Email us: editor@actascientific.com

Contact us: +91 9182824667

Citation: Mario Renato Papa-Vettorazzi., et al. "Results of Combined Small Incision Cataract Surgery and Cyclodialysis in Low Income Patients with Glaucoma and Cataract". Acta Scientific Ophthalmology 5.1 (2022): 04-12. 